\title{
Numerical study of transport in a dissipative medium
}

\author{
P. M. V. B. Barone \\ Instituto de Física "Gleb Wataghin," Universidade Estadual de Campinas, Caixa Postal 6165, \\ 13081 Campinas, São Paulo, Brazil \\ and Departamento de Física, Universidade Federal de Juiz de Fora, 36035 Juiz de Fora, Minas Gerais, Brazil \\ C. Morais Smith \\ Instituto de Física “Gleb Wataghin," Universidade Estadual de Campinas, Caixa Postal 6165, \\ 13081 Campinas, São Paulo, Brazil \\ and Departamento de Física, Universidade Estadual Paulista, 17033 Bauru, São Paulo, Brazil \\ D. S. Galvão \\ Instituto de Física "Gleb Wataghin," Universidade Estadual de Campinas, Caixa Postal 6165, \\ 13081 Campinas, São Paulo, Brazil \\ (Received 7 October 1991)
}

\begin{abstract}
A numerical study of propagation of a particle through a one-dimensional dissipative medium is presented. The medium is composed of several dissipative sections, which are characterized by their friction coefficients $\eta$. In particular, we have considered two types of friction coefficients distributed orderly or disorderly along the chain. For the same relative proportion of the coefficients, we have found that transport can be enhanced in the disordered distribution in comparison with the ordered one. We also show how this can be considered an approximated way to treat the propagation in a dissipative medium with a position-dependent friction coefficient.
\end{abstract}

PACS number(s): 05.40. $+\mathrm{j}, 05.60 .+\mathrm{w}$

\section{INTRODUCTION}

The dynamics of a dissipative particle has been exhaustively studied for several years. The most successful model was introduced by Caldeira and Leggett (CL) [1] in order to treat quantum Brownian motion. Their approach consists in coupling the Brownian particle to a bath of harmonic oscillators and then applying canonical quantization to the composite system. After this, the Feynman-Vernon procedure [2] is employed in order to find the reduced dynamics of the particle.

Some essential features of the CL approach are the spectral density of the bath, the initial condition, and the form of the coupling between particle and reservoir. The specific choice made by CL for these issues was motivated by the physics of the system studied by them, namely, the magnetic flux in superconducting quantum interference devices (SQUID's) [1].

Later on, new applications to other systems have required generalizations on these points. Thus, the socalled Ohmic dissipation employed by $\mathrm{CL}$ has been modified to treat both sub-Ohmic and super-Ohmic cases [3-5]. Also, the factorable initial condition of CL was extended by other authors [6,7]. More recently, the form of the coupling was modified from coordinate-coordinate coupling to momentum coupling [8].

All these approaches have been applied in a series of problems involving systems coupled to some kind of environment, such as electron transfer in chemical and biological processes [9], diffusion of muons in metals [10], ra- diation damping [4], and polarons [11].

Here we are interested in another kind of problem. We study the propagation of a particle through a onedimensional dissipative medium divided in sections, each one with different properties. This means that each section has a distinct bath of harmonic oscillators and the coupling differs from section to section. We treat only two types of dissipative sections, allowing several relative concentrations and imposing ordered and disordered distributions. The question we want to answer is, given an input energy for an incoming particle in one extremity of the dissipative chain, what will be the output energy in the other end?

The full quantum-mechanical treatment of this problem involves investigating in detail the transition from one section to another. At the interface between two sections there may arise a reflected wave which must be taken into account. However, we have only considered a "classical limit" of the problem where these effects were neglected.

Furthermore, it is clear that this model of a dissipative chain is conceptually distinct from the other onedimensional models currently studied in the literature [12]. While the dissipative chain involves nonelastic processes, the tight-binding model or a sequence of potential barriers involves only elastic interactions with the traveling particle. Thus our results have no direct relation with the results previously obtained with the later models [12].

In Sec. II we present the model and briefly discuss 
some of its features. The results obtained for ordered and disordered dissipative chains are shown and discussed in Sec. III. Finally, in Sec. IV we present a summary and the final remarks.

\section{MODEL}

We consider a one-dimensional system described by the following Hamiltonian:

$$
\begin{gathered}
H=\frac{p^{2}}{2 m}+V(q)+\sum_{i} s_{i}\left[\sum_{k}\left[\frac{p_{k i}^{2}}{2 m_{k i}}+\frac{1}{2} m_{k i} \omega_{k i}^{2} q_{k i}^{2}\right]\right. \\
\left.+\sum_{k} a_{k i} q_{k i} q\right],
\end{gathered}
$$

where $q, p$, and $m$ are the coordinate, the momentum, and the mass of the particle and $q_{k i}, p_{k i}, m_{k i}$, and $\omega_{k i}$ are the coordinate, the momentum, the mass, and the frequency of the $k$ th oscillator of the $i$ th section. $V(q)$ is an external potential, $a_{k i}$ is a coupling constant, and $s_{i}$ switches on the bath corresponding to each section when the particle is in it. Its value is 1 when the particle is inside the $i$ th section, and zero otherwise. Thus the interaction is suddenly switched when the particle goes into the section, in such a way that the initial condition is factorable.

The parameters in Eq. (1) need be known only in a particular combination, by means of a spectral density function $J(\omega)$, defined as

$$
J(\omega)=\frac{\pi}{2} \sum_{k} \frac{a_{k}^{2}}{m_{k} \omega_{k}} \delta\left(\omega-\omega_{k}\right) .
$$

The specific form of this function depends on the particular system under consideration either through its microscopic features or through its equation of motion in the classical limit [3]. We restrict ourselves to the case of Ohmic dissipation, where the equation of motion in the classical limit is Langevin's equation [1]. In this case, the spectral density behaves as $J(\omega)=\eta \omega \Theta(\omega-\Omega)$, where $\eta$ is the dissipation constant in Langevin's equation, $\Omega$ is a cutoff frequency, much higher than the characteristic frequencies involved in the problem under study, and $\theta$ is the Heaviside function. Thus, the coupling between the particle and the bath in the $i$ th section, which is controlled by the parameters $a_{k i}$, could equivalently be represented by the dissipation constant $\eta_{i}$.

We study here the case of zero external potential $V(q)=0$ giving an input energy to the free Brownian particle when it impinges on one end of the chain and comparing the output energy in the opposite side, both in an ordered and in a disordered chain. The disorder is introduced by building a random sequence of dissipation constants.

The particle will be represented by a Gaussian wave packet centered around $\bar{q}$ and with a mean momentum $\bar{p}$. These variables have a time evolution given by [1]

$$
\begin{aligned}
& \bar{q}(t)=\frac{\sinh \gamma t}{m \gamma \exp (\gamma t)} \bar{p}(0)+\bar{q}(0), \\
& \bar{p}(t)=\exp (-2 \gamma t) \bar{p}(0),
\end{aligned}
$$

where $\gamma=\eta / 2 m$.

In the following we restrict our study to the behavior of these mean values. This "classical limit" neglects quantum effects on the interfaces of the sections. This point will be discussed in detail later. We further impose the continuity of the dynamical variables on the boundaries of the sections. Thus, for each section, with a given input energy, we obtain the output energy from Eqs. (3) and (4) and the additional condition $\bar{q}(t)-\bar{q}(0)=l$, where $l$ is the length of the sections.

\section{RESULTS AND DISCUSSIONS}

The presence of dissipation makes the evolution of the particle in a particular section of the chain strongly dependent on its entire previous history. Because of this memory effect, an analytical study based on the simple equations (3) and (4) becomes very hard. In fact, iterating Eq. (4) over the extreme points of the sections, $i=1,2, \ldots, N$, gives

$$
\bar{p}_{N}=\exp \left[-2 \sum_{i=1}^{N} \gamma_{i} t_{i}\right) \bar{p}_{0},
$$

where $t_{i}$ is the time spent in the $i$ th section, $\bar{p}_{0}$ is the mean momentum of the incoming wave packet, and $\bar{p}_{N}$ is the mean value after $N$ sections. It is easy to see that $t_{i}$ is strongly dependent on all the previous $t_{i-1}, t_{i-2}$, etc., and thus the same happens with $\bar{p}_{N}$.

On the other hand, Eq. (5) allows us to define an effective dissipation constant for the chain

$$
\Gamma=\frac{\sum_{i=1}^{N} \gamma_{i} t_{i}}{\sum_{i=1}^{N} t_{i}},
$$

which suggests examining the problem from a different point of view. We can think about a particle diffusing through an inhomogeneous dissipative medium, in the present case with a piecewise-constant friction coefficient. That is, we can look at the medium from a global point of view and consider the variation of the friction coefficient as a position dependence of $\eta$. We will return to this point below.

We now proceed to the numerical calculations. In order to determine specific values for the dissipation constants, we have separately studied homogeneous chains composed uniquely by one kind of section. The value for the corresponding $\eta$ is obtained from the conditions: (i) weak dissipation or, equivalently, nearly free transport (a-type section) and (ii) strong dissipation or large energy loss, about $85 \%$ of the input energy ( $b$-type section). We allow the concentration of $b$-type sections to vary from $5 \%$ up to $50 \%$. In all calculations we have used chains with a fixed number of 1000 sections.

Many distinct chain configurations with random distributions of $b$-type sections were carried out for each concentration. We have observed a sensitivity of the output energy with the fluctuations of the concentration along the chain portions around the overall value. In particu- 
lar, the largest output energies were obtained when the concentration of $b$-type sections was smaller in the initial portions of the chain than the overall value. This can be checked by observing the momentum evolution section to section of the chain. Table I shows the output energy of some random distributions of $b$-type sections and of the ordered sequence $a b a b \ldots$ for a $50 \%$ concentration.

It is interesting to see that transport along the chain is favored in some configurations with random distributions in comparison with the ordered one. The physical meaning of disorder here is quite different from the case of interference of wave functions. There are no coherence effects in the present classical calculations. While the particle is diffusing from section to section, the rate of energy loss is proportional to $\eta \dot{\bar{q}}^{2}$, which is clearly higher in the beginning of the propagation. Thus, these results can be viewed as a consequence of the irreversibility of the dissipation process and the local fluctuations of the concentration.

We also compare particular random distributions (all produced by the same random-number generator) of $b$ type sections with ordered distributions for several concentrations. The results are shown in Fig. 1. For all concentrations in the range 5-50\%, transport is enhanced in the disordered chains. For instance, in the case of $10 \%$ concentration of $b$-type sections, if they are randomly distributed, the output energy is higher than it is for the ordered distribution. Although this cannot be generalized to all the possible random distributions, due to the strong influence of memory effects, it is remarkable that it can happen in some of them. So for different concentrations and configurations, we can conclude that the diffusion through a disordered chain could be easier than through an ordered one.

We have mentioned above the possibility of changing the point of view to use the model to treat classical diffusion through one-dimensional inhomogeneous dissipative media. We can see the dissipative chain as a medium with a position-dependent dissipation coefficient with values

$$
\eta(q)= \begin{cases}\eta_{a}, & q \in a \text {-type section } \\ \eta_{b}, & q \in b \text {-type section }\end{cases}
$$

TABLE I. The output energy of some random distributions and the ordered $a b a b \ldots$ distribution in the case of a $50 \%$ concentration. The units are arbitrary and the input values are the same as in Fig. 1.

\begin{tabular}{lc}
\hline \hline Distribution & Output energy \\
\hline random 1 & 2693.5 \\
random 2 & 2726.1 \\
random 3 & 2775.4 \\
random 4 & 2865.6 \\
random 5 & 2880.0 \\
random 6 & 2911.0 \\
ordered & 2815.6 \\
\hline \hline
\end{tabular}

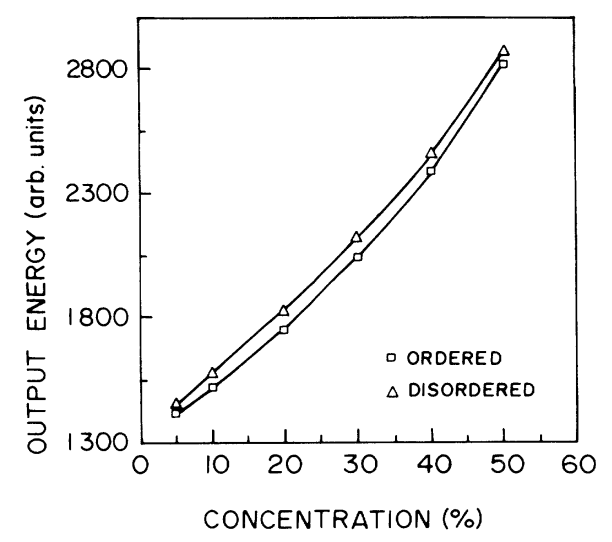

FIG. 1. Output energy vs concentration of $b$-type sections. The values in the graph were calculated for $E_{\text {in }}=8000, m=0.1$, $\hbar=1$, and $l=20$ (arbitrary units), using the same randomnumber generator.

This piecewise-constant function can be extended to many possible values $\eta_{1}, \eta_{2}, \ldots, \eta_{n}$, at the same time that the length of the sections is reduced, to furnish a discrete sample of an ordinary function. With this process we can approximate the case of position-dependent $\eta(q)$ and define the effective dissipation constant as

$$
\Gamma^{\prime}=\lim _{l \rightarrow 0} \Gamma=\frac{1}{T} \int_{0}^{L} \frac{\eta(q)}{\dot{q}} d q
$$

where $L$ is the total length of the medium. Then the evolution of the mean value of the momentum will be given by an expression similar to (5) (with an integral replacing the summation) regarding the medium from a global point of view.

This approximation to the real problem of positiondependent dissipation involves the assumption of a distinct bath of oscillators for each small length $l$ and the possibility of artificial effects due to the sudden switch of the correspondent interactions. However, as far as we are concerned with the mean values, these effects do not make any difference, once the mean values are the same for both the uncoupled and the coupled initial conditions $[6,7]$.

On the other hand, the full quantum-mechanical problem will be affected not only by the artificial initial condition but also by the possible reflection on the interfaces of the sections. Also, the matching of $\bar{q}$ and $\bar{p}$ values at each interface is by itself a classical ingredient which cannot be retained in order to treat the problem quantum mechanically.

The energy range we have employed in our numerical study is such that the width of the wave packet, $\sigma$, is always much less than the length of the sections, say, $\sigma / l<10^{-3}$. Thus we can justify the use of only a classical limit, but quantum effects must be taken into account in order to provide complete information about transport properties of the dissipative chain. 


\section{SUMMARY AND FINAL REMARKS}

We have presented a study of transport through a dissipative medium composed of an array of sections with different friction coefficients. We have treated dissipation by means of the Caldeira-Leggett approach. Numerical calculations of a classical limit of the problem allow us to conclude that propagation through the dissipative chain is strongly affected by the past history, as a consequence of the irreversibility of dissipation.

In the special case of two types of dissipative sections, we found that their disordered distribution can favor the propagation when compared to an ordered distribution. This is not a question of coherence but a classical phenomenon. Thus this model has a classical contribution for transport beside its possible quantum contributions, which were not considered here. Furthermore, the classical version of a position-dependent dissipative medium can be described by means of our approach, although new questions have to be addressed.

Finally, we pointed out some features that remain to be answered if we want to solve the problem quantum mechanically, which include the calculation of reflection coefficients for a wave packet in the interfaces of two dissipative sections or even when the particle goes from a nondissipative to a dissipative medium.

\section{ACKNOWLEDGMENTS}

The authors wish to thank Professor A. O. Caldeira and A. H. Castro Neto for many fruitful discussions. The authors also thank the Brazilian Agencies $\mathrm{CNPq}$, FAPESP, and CAPES for financial support.
[1] A. O. Caldeira and A. J. Leggett, Phys. Rev. Lett. 46, 211 (1981); Ann. Phys. (N.Y.) 149, 374 (1983); 153, 455 (E) (1984).

[2] R. P. Feynman and F. L. Vernon, Jr., Ann. Phys. (N.Y.) 24, 118 (1963).

[3] A. J. Leggett, S. Chakravarty, A. T. Dorsey, M. P. A. Fisher, A. Garg, and W. Zweger, Rev. Mod. Phys. 59, 1 (1987).

[4] P. M. V. B. Barone and A. O. Caldeira, Phys. Rev. A 43, 57 (1991).

[5] H. Grabert, P. Schramm, and G.-L. Ingold, Phys. Rep. 168, 115 (1988).

[6] V. Hakim and V. Ambegaokar, Phys. Rev. A 32, 423
(1985).

[7] C. Morais Smith and A. O. Caldeira, Phys. Rev. A 36, 3509 (1987); 41, 3103 (1990).

[8] A. H. Castro Neto and A. O. Caldeira, Phys. Rev. Lett. 67, 1960 (1991).

[9] A. Garg, J. N. Onuchic, and V. Ambegaokar, J. Chem. Phys. 83, 4491 (1985); J. N. Onuchic, D. N. Beratan, and J. J. Hopfield, J. Phys. Chem. 90, 3707 (1986); J. N. Onuchic, ibid. 86, 3925 (1987).

[10] P. Hedegard, Phys. Rev. B 35, 533 (1987); 35, 6127 (1987).

[11] A. H. Castro Neto and A. O. Caldeira (unpublished).

[12] P. Erdos and R. C. Herndon, Adv. Phys. 31, 65 (1982). 